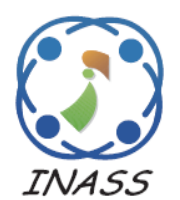

\title{
BER Comparison and Enhancement of Different Optical OFDM for VLC
}

\author{
Samir M. Hameed ${ }^{1 *}$ \\ Sinan M. Abdulsatar ${ }^{2}$ \\ Atheer A. Sabri ${ }^{3}$ \\ ${ }^{1}$ University of Information Technology and Communications, Baghdad, Iraq \\ ${ }^{2}$ Electrical Engineering Department, University of Technology-Iraq, Baghdad, Iraq \\ ${ }^{3}$ Communication Engineering Department, University of Technology-Iraq, Baghdad, Iraq \\ * Corresponding author's Email: samirmhameed@uoitc.edu.iq
}

\begin{abstract}
Visible Light Communication (VLC) becomes a promising technology in the Optical Wireless Communication OWC field. VLC has been developed based on Optical Orthogonal Frequency Division Multiplexing $(\mathrm{O}-\mathrm{OFDM})$ in recent years. The Bit Error Rate (BER) performance for various O-OFDM is studied in this paper. The non-linearity characteristic of Light-Emitting Diode (LED), modulation constellation size, O-OFDM scheme, and channel types impacts the BER performance of VLC systems significantly. The proposed model utilizes the conVolutional encoder of 2/3 rate and seven memory cells in the transmitter with O-OFDM for BER enhancement. At the receiver, the Viterbi algorithm system is employed with hard or soft-decision decoding. The proposed system is evaluated in Line-of-Sight and Non-Line-of-Sight (NLOS) channels. In the NLOS model, Zero Forcing Equalizer (ZFE) with channel estimation is added at the receiver path for equalizing the multipath diffused components. This study proves the capability of the proposed system in mitigating the LED non-linearity and reducing BER. The simulation results show the Signal-to-Noise Ratio (SNR) enhancement of the exanimated O-OFDM-based softdecision between 7 to $10 \mathrm{~dB}$ for the BER 10-5 in the LOS model, and for the NLOS model, the BER can be improved from 0.02 up to $10-5$ at SNR $45 \mathrm{~dB}$.
\end{abstract}

Keywords: O-OFDM, ACO-OFDM, DCO-OFDM, ADO-OFDM, ConVolutional coding, Viterbi decoder, VLC.

\section{Introduction}

Optical Wireless Communication (OWC) is a modern communication that uses light as the carrier of information instead of the Radio Frequency (RF); it supports a high data rate communication with extremely secure links [1-5]. Visible Light Communication (VLC) is a type of OWC that uses visible light in data transmission used in many applications [1, 3, 5, 6]. VLC uses Light Emitting Diode (LED) in the transmitter and photo-sensing such as Photodiode (PD) in the receiver [5]. Intensity Modulation with Direct Detection (IM/DD) is a helpful technique used with VLC [7]. The Optical Orthogonal frequency-Division Multiplexing (OOFDM) has widely used in IM/DD systems for the advantages of high data rates transfer, compatible with multicarrier modulation, and symbol interference resistance [5, 7-9]. The IM/DD systems require positive real positive signals for data transmission by LED. The O-OFDM produces real positive values that differ from OFDM used in RF systems that deal with complex bipolar signals [10]. Many O-OFDM types are proposed depending on generating non-negative values, and Hermitian symmetry is used to convert complex values to real [10-12]. In DC Biased Optical OFDM (DCO-OFDM), the DC bias values are added to the generating OFDM signals to produce positive symbols [13]. Asymmetrically Clipped Optical OFDM (ACOOFDM) uses only odd subcarriers for transmission and clipping the OFDM signals to zero for creating non-negative OFDM without affecting the information $[8,10]$. The optical power of DCOOFDM is ineffective due to DC bias, while ACOOFDM is better, but it has low spectral efficiency [11]. In Asymmetrically Clipped DC Biased Optical OFDM (ADO-OFDM) is an enhanced combining technique of both DCO-OFDM and ACO-OFDM [10, 
13]. ADO-OFDM has the same spectral efficiency as DCO-OFDM, but it has better optical power efficiency [14]. The Bit Error Rate (BER) of ACOOFD is better than ADO and DCO-OFDM because it is independent of DC bias, where the DC bias value increases BER [15]. In [10], the authors presented the performance enhancement model for various OOFDM based on the minimum mean square error equalizer and Hamming codes. In [16], BER comparisons were made for both Line-of-Sight (LOS) and Non-Line-of-Sight (NLOS) channels based on ACO-OFDM, DCO-OFDM, and ADOOFDM. The simulation results were evaluated in [10], [16] by looking at the linear behavior characteristics of the LEDs. The LED's transfer function results in non-linearity because of the cut-off region for Voltages below the turn-on value and distorts the maximum allowable signals [17]. The LED nonlinearity will impact the performance of VLC systems based on O-OFDM and lead to BER degrading [18-20]. Furthermore, many other influencing parameters affect BER performance, such as modulation order, Signal-to-Noise Ratio (SNR), channel types, and OFDM schemes [10, 13, $16,20,21]$.

In this work, the BER performance of the VLC systems for different O-OFDM is examined and simulated. The simulation considers the LED nonlinearity for LOS and NLOS channels. The Rapps model is used for modeling the output current (I) to input Voltage (V) characteristic of LED, which describes the LED non-linearity [12-15]. The BER enhancement for VLC systems is proposed using a conVolutional encoder at the transmitter and the Vetribi decoder at the receiver. Also, the proposed system is considered to mitigate the LED nonlinearity, noise occurring in O-OFDM due to the clipping process. The organization of the remainder of this paper as follows. In section 2, the system model is described. The proposed BER enhancement model has presented in section 3 . In section 4 , the simulation results are discussed. Finally, the conclusions are presented in section 5 .

\section{System model}

In this section, the system model of VLC for three O-OFDM types is described, DCO-OFDM, ACOOFDM, and ADO-OFDM. The OWC channel effect is briefly viewed for both LOS and NLOS cases. The Rapps model is discussed in this section for modeling the non-linearity of LED.

\subsection{The O-OFDM model}

The general block diagram for the transmitter OOFDM system model of the VLC is shown in Fig.1.a. The information is modulated by MultilevelQuadrature Amplitude Modulation (M-QAM); the resulting modulated signals are composed of complex symbols. Multicarrier modulation technology has been used to leverage high data rates, intersymbol resistance, the LED's non-linearity experience [22]. The Inverse Fast Fourier Transform (IFFT) is used after converted the modulated information from Serial to Parallel (S/P). The resulting output signal from IFFT is in the time domain; it described as [23]:

$$
X_{n}=\frac{1}{\sqrt{N}} \sum_{k=0}^{N-1} X_{k} e^{\frac{i 2 \pi n k}{N}} \text { for } 0 \leq n \leq N-1
$$

where $\mathrm{N}$ is the number of the subcarriers, $\mathrm{X}_{\mathrm{k}}$ is the frequency domain data. The Hermitian symmetry transform is used with O-OFDM to produce real-time domain symbols as [23]:

$$
X_{N-k}=X_{k}^{*}
$$

where * refer to the conjugate value, the Cyclic Prefix (CP) will be added to $X_{n}$ after converting the symbols from Parallel to Serial $(\mathrm{P} / \mathrm{S})$ as a guard interval for intersymbol interference elimination [21]. In DCO-OFDM, half of the subcarriers are used for modulation, and the non-negative values can be obtained by adding a DC bias [13]. The additional DC bias adds clipping noise at the transmitter that distorts the received signal in the DCO-OFDM and decreases BER and optical power efficiency $[14,16]$. The ACO-OFDM overcomes the DC bias issue by transmitting data symbols via only the odd subcarriers, and all the even subcarriers are set to zero [8]. The non-negative values are obtained by clipping the time domain signals to zero in even subcarriers without affecting the transmitted data [21]. The signal clipping to zero creates clipping noise in even subcarriers [11]. The BER performance and optical power efficiency of ACO-OFDM are better than DCO-OFDM due to the simplicity of data recovery, which is only limited to the odd subcarriers [14]. The ADO-OFDM uses a transmission combination of both ACO-OFDM and DCO-OFDM by extracting the benefits of both technologies. The odd subcarriers are reserved for ACO-OFDM, while the even subcarriers for DCO-OFDM [16]. Although ADOOFDM is classified as an enhanced O-OFDM technique, it suffers from DC bias in the even subcarriers, which leads to distortion of the signals 


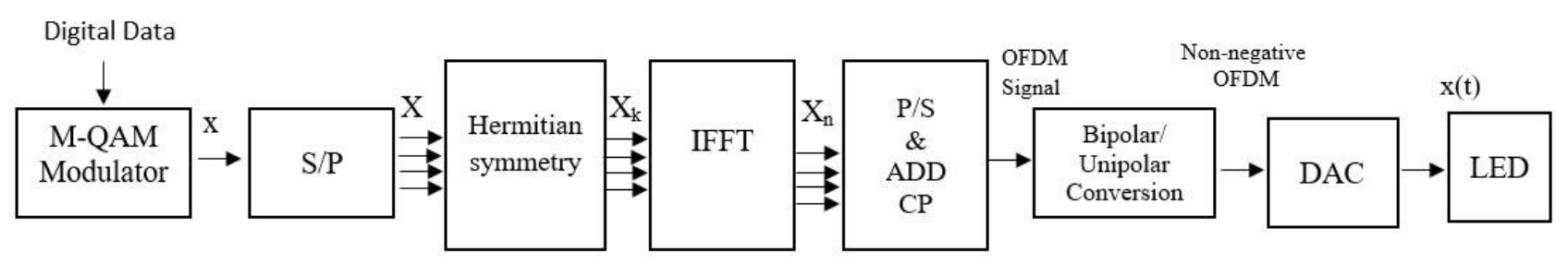

(a)

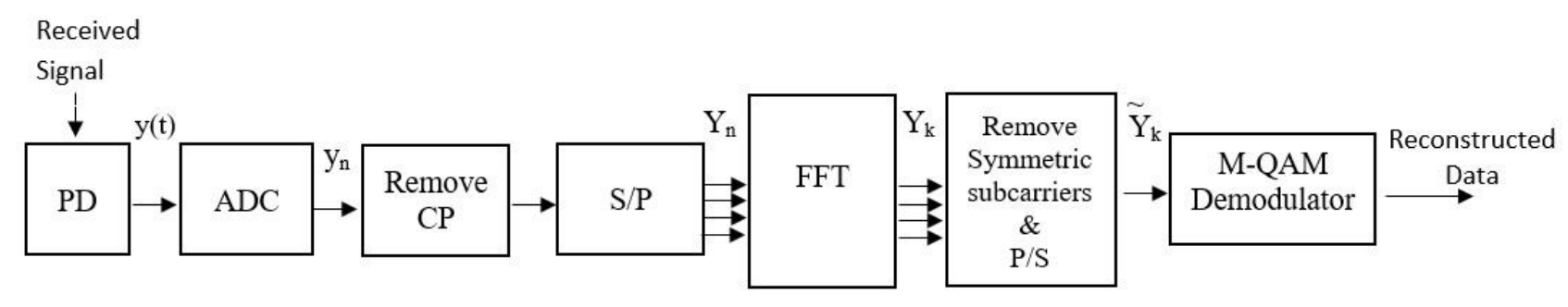

(b)

Figure. 1 General O-OFDM system model (a) transmitter (b) receiver

and an increase in the BER [14]. The number of data enabling subcarriers of ADO-OFDM is similar to DCO-OFDM, which uses only half of the actual subcarriers due to Hermitian symmetry. In contrast, ACO-OFDM uses only a quarter of the total subcarriers. The Digital-to-Analogue Converter (DAC) is used to convert the O-OFDM symbols to analog for intensity modulation process by LED. The transmitted intensity is received at the receiver by PD and converted to an electrical signal. The signal is converted to digital for signal processing by Analogue-to-Digital (ADC), as mentioned in Fig. 1.b, which illustrates the general O-OFDM receiver. The received signal is transformed to the frequency domain using Fast Fourier Transform (FFT) after CP is removed and converted to parallel form; it described as [23]:

$$
Y_{k}=\frac{1}{\sqrt{N}} \sum_{n=0}^{N-1} Y_{n} e^{\frac{-i 2 \pi n k}{N}} \text { for } 0 \leq k \leq N-1
$$

where $Y_{k}$ is the frequency domain data of the received signal. The demodulation process is accomplished after assigning the allocated subcarriers that carry data. The Additive White Gaussian Noise (AWGN) is added to the received signal by converting intensity from an electrical signal to an electrical signal at PD. The AWGN is a combination of thermal noise and shot noise; the received signal before ADC described as [21]:

$$
y(t)=h(t) \otimes x(t)+w(t)
$$

where $\mathrm{h}(\mathrm{t})$ is the impulse response of the OWC channel, $\otimes$ is the conVolution process, $x(t)$ is the transmitted signal, and $\mathrm{w}(\mathrm{t})$ is AWGN. In VLC systems, there are two types of indoor channel, LOS and NLOS [24]. The VLC's LOS channel follows the Lambertian pattern model of indoor OWC [16]. The impulse response for the NLOS channel contains a direct way LOS and diffuse components due to reflection and refraction of lights across the surfaces [16], [24]. The received signal after ADC can be written as in discrete form:

$$
y_{n}=h_{n} * x_{n}+w_{n}
$$

where $*$ is discrete conVolution and refers to the discrete form. Eq. (5) can be re-written in the frequency domain after FFT as [21]:

$$
Y_{k}=H_{k} X_{k}+W_{k}
$$

where $\mathrm{H}_{\mathrm{k}}$ is the frequency response of the OWC channel, $X_{k}$ is the FFT of the transmitted signal, and $\mathrm{W}_{\mathrm{k}}$ is the frequency response of AWGN. The data transmitted in NLOS is distorted by the multipath impulse response of the channel, so to recover the transmitted data from the received signal, the equalizer will be used to multiply the $\mathrm{Y}_{\mathrm{k}}$ by $1 / \mathrm{H}_{\mathrm{k}}$. The recovered transmitted signal can be described as [25]:

$$
\widetilde{X_{k}}=\frac{Y_{k}}{H_{k}}=X_{k}+\frac{W_{k}}{H_{k}}
$$




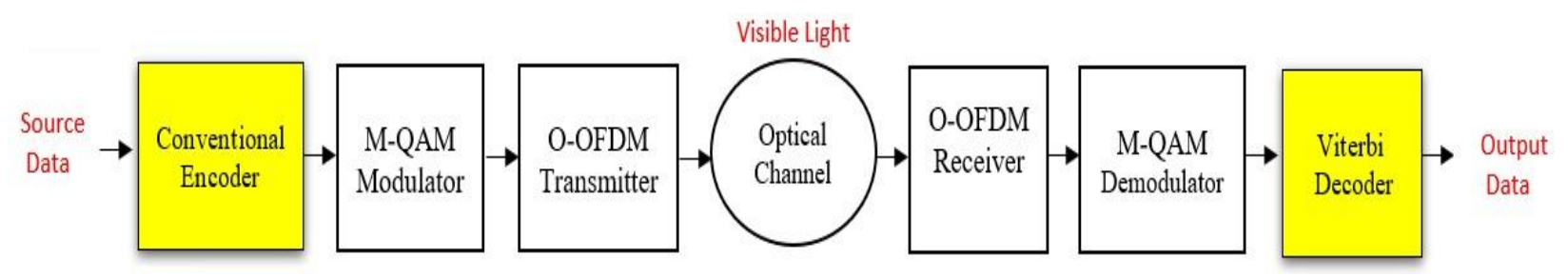

Figure.2 The proposed BER enhancement model for the LOS channel

The Zero Forcing Equalizer (ZFE) is used at the receiver to estimate the channel's frequency response and applies Eq. (7) for restoring the transmitted signal for each subcarrier

\subsection{Rapps model}

The LED's non-linearity effects on the performance of VLC can be described using the LED V-I characteristic. Rapps model, which is commonly used in RF systems, is used to define the behavior of LED [17-20, 26]. The LED model can be described as:

$$
I_{L E D}\left(V_{L E D}\right)= \begin{cases}\frac{f\left(V_{L E D}\right)}{\left(1+\left(\frac{f\left(V_{L E D}\right)}{I_{m}}\right)^{2 k}\right)^{\frac{1}{2 k}}} & \text { if } V_{L E D} \geq V_{T h} \\ 0 & \text { if } V_{L E D}<V_{T h}\end{cases}
$$

where $\mathrm{k}$ is knee factor described the non-linearity, $I_{m}$ is a maximum allowable current to pass through the LED, $f\left(V_{L E D}\right)$ is a function relating V-I characteristics, and $V_{T h}$ is the turn-on Voltage of the LED. In Eq. (8), The $V_{L E D}$ represents the O-OFDM signal of the VLC, which has real and unipolar values. The knee factor is either set to constrain the clipping hard or soft so that this factor can control the upper saturation of the V-I curve [18].

\section{The proposed BER enhancement model}

BER is the main factor for computing the OOFDM system's performance, and it is inversely proportional to the SNR. The LED non-linearity will increase BER and decreases the performance of the VLC system. The channel coding techniques are widely used for distortion compensation due to nonlinearity [21]. The proposed BER enhancement model for LOS is considered to use a conVolutional encoder before the M-QAM modulator of the OOFDM system at the transmitter. However, at the OOFDM receiver, the Viterbi algorithm is investigated after the demodulator as channel decoder, as shown in Fig.2. The proposed model achieves VLC's gain due to code insertion that improves BER performance.
Also, it mitigates the LED non-linearity and signals distortion arising from the clipping process in different O-OFDM schemes. ConVolutional code is a kind of error-correcting code that is easy to implement based on logic operations. It is a channel coding technology and is widely used in digital communication systems [15].

Moreover, it is a finite state machine consisting of K-stage shift registers; the output code $(n)$ combining from the input bits $(k)$ shifted across the registers, polynomial generator, and modulo- 2 adders for each coded bit [27, 28]. The general form describing the conVolutional encoder is $(n, k, \mathrm{~K})$, and the code rate is $k / n$; Fig. 3 illustrates an example of the encoder with $(2,1,3)$ and $1 / 2$ rate. The channel coding improves BER but reduces the spectral efficiency due to the extra redundant bits. Therefore, it must balance these two-parameter choosing the coding rates. Our proposed BER enhancement model is regarded to use $(3,2,7)$ conVolutional encoder with a $2 / 3$ rate as channel coding. The encoder consists of two segments: the upper one contains 4shift registers and 3-shift registers used for the lower path, as shown in Fig.4. The BER depends on the polynomial generator stating the output, the number of the memory registers used, and encoder rate.

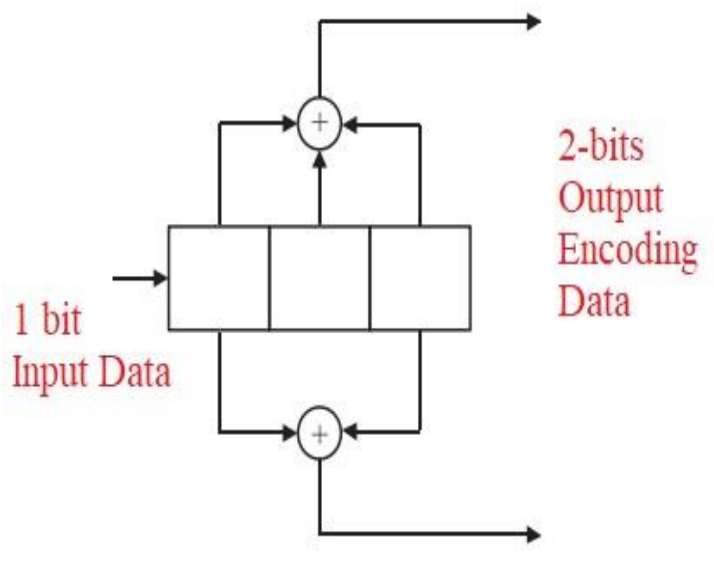

Figure.3 Example of $(2,1,3)$ conVolutional encoder 
Code performance can be enhanced by increasing the constraint length of the encoder instead of changing the coding rate. We select a constraint length of $(5,4)$ tested and performed well for the BER. The output code will initiate state transition based on the data input's current and previous state. This scheme reduces the error caused due to channel and LED non-linearity and is categorized as non-systematic code. The systematic code is the encoder output is a branch of input data; instead, the non-systematic code is not part of input data [28]. The non-systematic conVolutional code has a higher free distance so that it tends to be recommended over systematic code [27]. At the receiver, we use a Viterbi dynamical algorithm that computes the best match in decoding the conVolutional codes. The Viterbi decoder is a Maximum Likelihood Sequence Estimator (MLSE), which interprets the sequence based on the sequence probability maximization [27-29]. The maximization becomes similar to the minimum distance decision (d) as [27]:

$$
d=\sum_{i=0}^{n-1}\left(r_{i}-x_{i}\right)^{2}
$$

where $r_{i}, x_{i}$ is the received and transmitted signal for the $\mathrm{N}$ symbol, respectively. The Viterbi algorithm computes the distance between received data with each word for all state transitions [29]. The possible paths with minimum distance for future processing need to be saved among the paths that merge into a condition in the code transitions. In contrast, all other paths can be removed without affecting optimal decoding $[28,29]$. The Viterbi algorithm is used to reduce the errors that have been detected by choosing the most probable paths [30]. There are two methods of decoding: hard and soft decisions. The harddecision decodes the bits as 1 or 0 depending on the threshold value rather than the soft-decision decoder bits in the range of values [27]. The Hamming distance is computed for the hard-decision and Euclidean distance for the soft-decision in the Viterbi algorithm for short path calculation through the code's trellis [29]. Soft computing has an advantage over hard decision techniques; therefore, it improves channel coding [27]. In the NLOS channel, the BER performance very worst for all O-OFDM types and

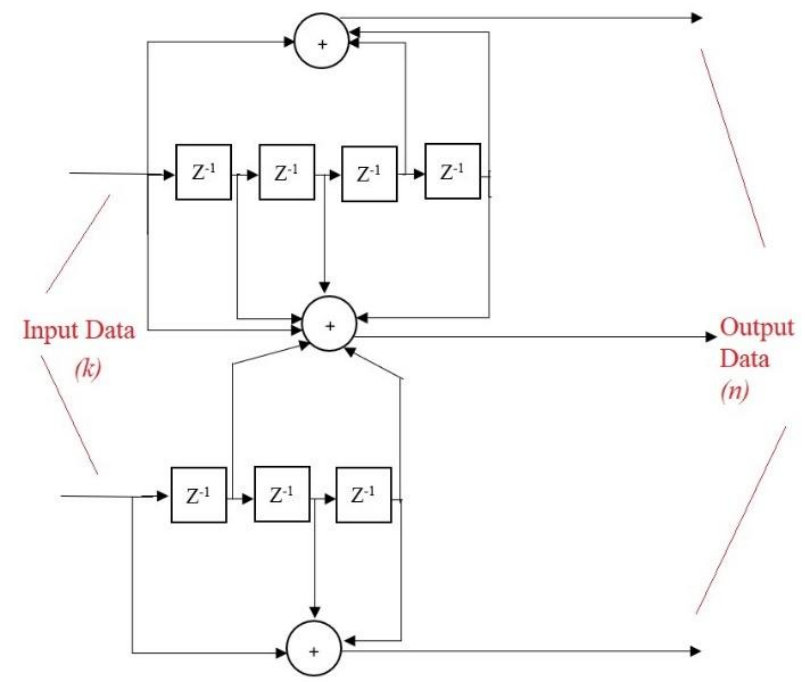

Figure. $4(3,2,7)$ ConVolutional encoder

any modulation constellation size due to the reflection and refraction of lights. The proposed BER enhancement model will be modified by adding channel estimation and a ZFE to avoid the signal's distortion due to slow fading.

The training sequence is predefined at the transmitter and receiver used to aid in channel estimation and is known as a pilot. There are two types of pilot used for insertion in channel estimation, block-type and combo-type [31]. Pilot signals of block-type inserted for all subcarriers of a single OFDM symbol, while pilots are placed at defined subcarriers [31, 32]. The block-type arrangement is preferred for VLC due to slow fading [32]. Fig. 5 presents the BER enhancement model for NLOS. The block type pilot arrangement is applied for this model; the channel estimation based on pilots can be described as [31]:

$$
H_{p}=\frac{Y_{p}}{X_{p}}
$$

where $\mathrm{X}_{\mathrm{p}}$ is the transmitted signal and $\mathrm{Y}_{\mathrm{p}}$ is the received signal for training signals. ZFE applies the inverse of Eq. (10) for the received signals to reconstruct the transmitted signal. Using a conVolutional encoder and Viterbi decoder with ZFE and channel estimation will increase the NLOS-VLC systems' performance by enhancing the BER.

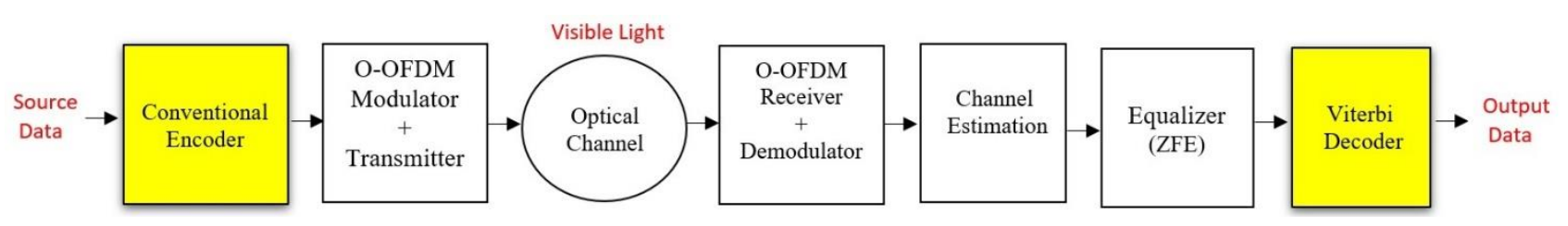

Figure. 5 The proposed BER enhancement model for NLOS channel 


\section{The simulation results}

BER simulations for DCO-OFDM, ACO-OFDM, and ADO-OFDM for different QAM constellation sizes are performed using Matlab R2019b software in this section. Simulation parameters are implemented for O-OFDM systems with IFFT / FFT 1024 subcarriers, and $\mathrm{CP}$ length is 32 . The $\mathrm{DC}$ biased value used for simulation is $0.25 \mathrm{~V}$ for DCO-OFDM and $0.15 \mathrm{~V}$ for ADO-OFDM. The non-linearity effect of LED is studied based on the Rappes model. Fig.6 presents different O-OFDM simulations in LOSVLC systems for LED in linear and non-linear states. These curves are accomplished for modulation of 256QAM for each subcarrier. The normalizing value of $f\left(V_{L E D}\right)=\frac{V_{L E D}}{R}$ in Eq. (8) is applied for modeling $\mathrm{I}_{\mathrm{LED}}\left(\mathrm{V}_{\mathrm{LED}}\right)$ with normlization resistance $\mathrm{R}$ equal to $1 \Omega[18,20] . V_{T h}$ is considered to be zero, $\mathrm{I}_{\mathrm{m}}=0.5 \mathrm{~A}$, and different values of knee factors $\mathrm{k}$ are tested. The results indicates that BER performance is inflenuced by the knee factor besides the noise. The BER performance will be decreased for small values of $k$, and the non-linearity has been increased, while at a high value for $\mathrm{k}$ such as 50, the LED behavior becomes nearly linear. In the case of NLOS-VLC, the BER performance gets worse, so channel equalization is required. Fig.7 shows the effects of knee factor for 256QAM modulation on the BER performance of various O-OFDM in NLOS after using the channel estimator-based block-type pilots with ZFE. It is assumed that the LED non-linearity is affected in NLOS more than in LOS, but the channel estimation and equalization have a good rule in $\mathrm{O}-$ OFDM systems for compensation this response. In this scheme, the pilot symbols are inserted for the first transmitting OFDM symbols transmitted. The frequency response of the channel is computed using Eq. (10). We propose modification for the channel estimation by tunning the system to recalculate the channel frequency response many times for investigating the channel response every 50 OFDM symbols. The performance will be improved by using the proposed model for various O-OFDM VLC systems. However, the conVolutional encoder and Viterbi decoder enhance the BER. Fig 8 illustrates the performance of DCO-OFDM, ACO-OFDM, and ADO-OFDM over the AWGN channel, 256QAM modulation, and the assumption of LED's linear behavior. The LOS and NLOS BER enhancement models use a conVolutional encoder that states in Fig. 8 with $2 / 3$ rate and seven memory cells. Both soft and hard decision forms are performed in the decoding process. The simulation results show the soft-decision decoding performance is better than the

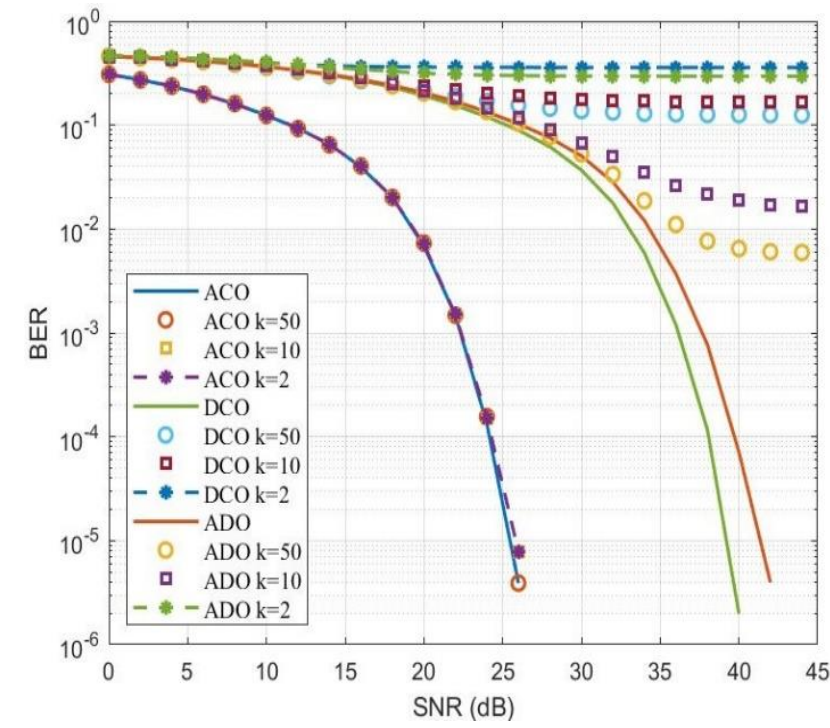

Figure. 6 BER in LOS for different O-OFDM

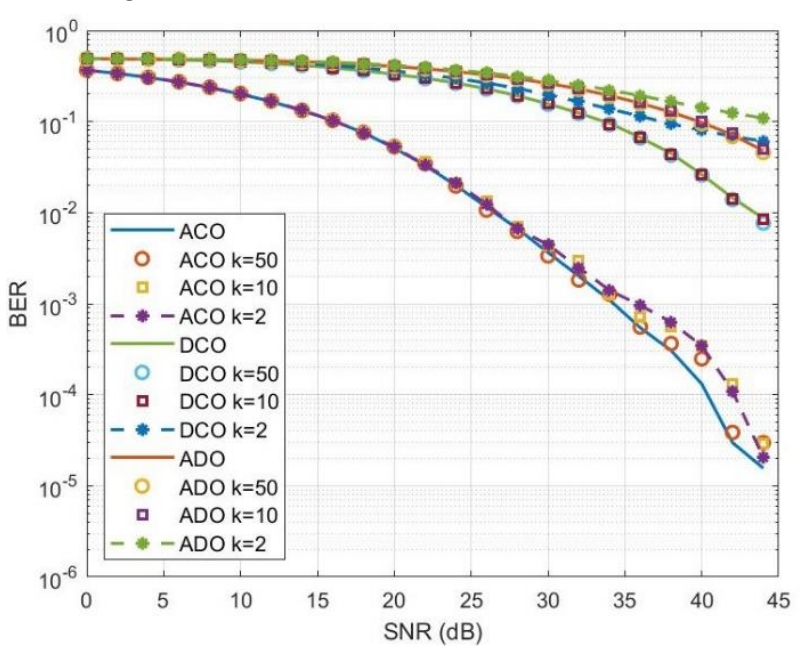

Figure. 7 BER in NLOS for different O-OFDM

hard-decision in all examined O-OFDM schemes. The BER performance for ADO-OFDM is beter than DCO-OFDM and ACO-OFDM has the best. Figs 9, 10 present DCO-OFDM, ACO-OFDM, and ADOOFDM BER performance in the case for the nonlinear state of the LED for $\mathrm{k}=2$. The comparisons between uncoded versus hard and soft decision coded O-OFDM for the proposed techniques in LOS and NLOS.

Two types of modulation, QAM and 256QAM, are chosen to study the effect of modulation order on BER performance. It is noticeable that the BER performance decreases for the larger modulation constellation size, and it requires higher values of SNR to reach lower values of BER. The better BER performance can be achieved using the proposed model, which successfully evaluates the LED's nonlinearity. The BER performance for ACO-OFDM is better, but it has a lower data rate, while the enhancement model gives a good result for ADO- 
OFDM over the DCO. The BER of the NLOS channel has higher than LOS due to multipath reflections. Table. 1 shows general comparisons between BER performance for DCO-OFDM, ACO-

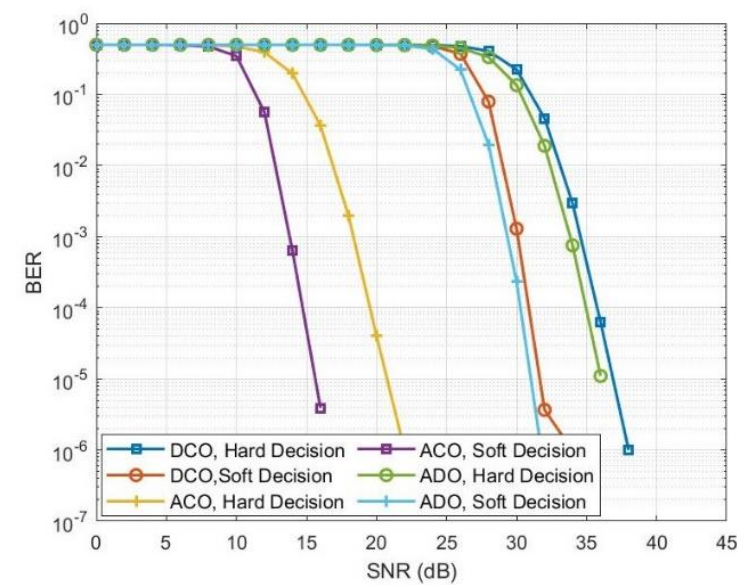

(a)
OFDM, and ADO-OFDM over 256QAM modulation. The O-OFDM models in [16] are used to generate uncoded responses compared with the proposed model.

Figure. 8 BER performance for different O-OFDM:

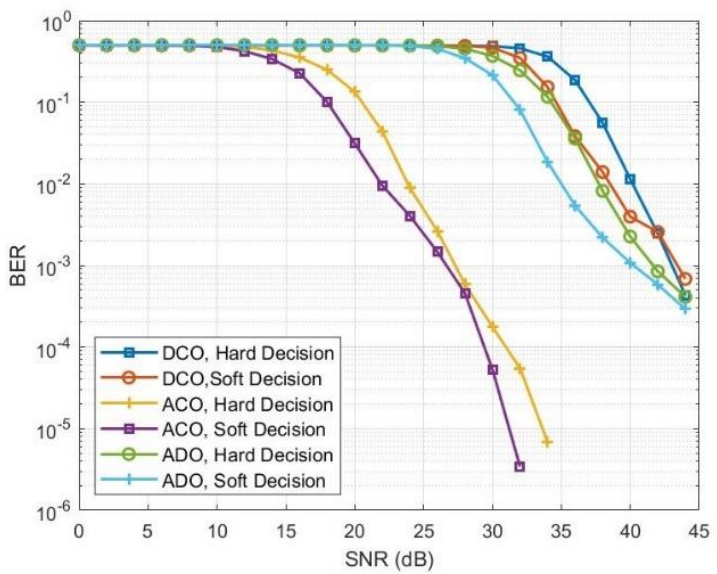

(b)

(a) LOS (b) NLOS

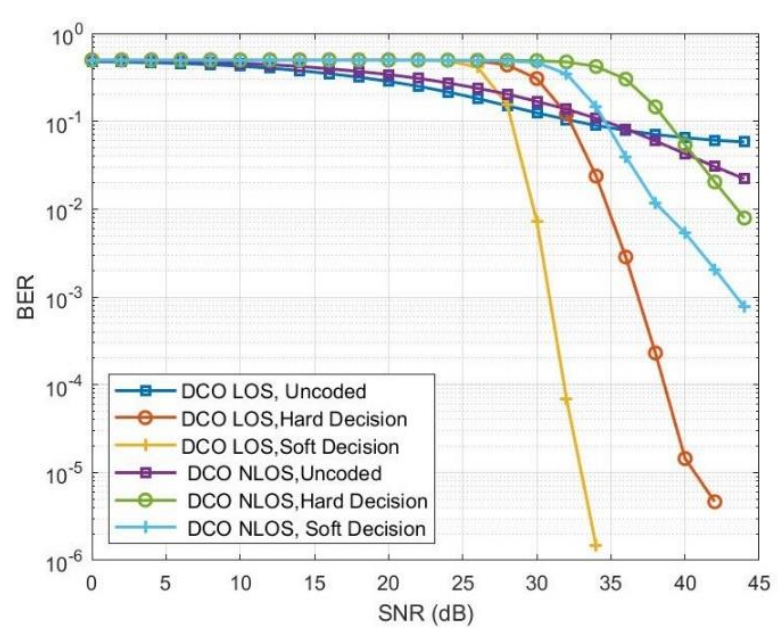

(a)

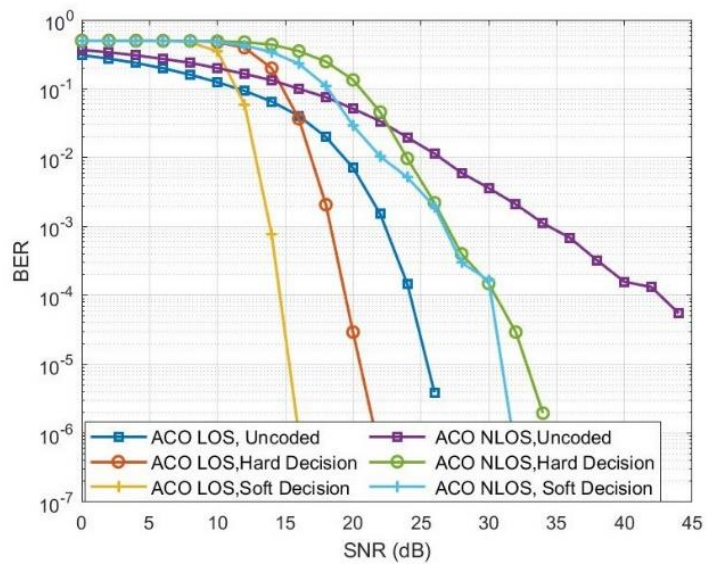

(b)

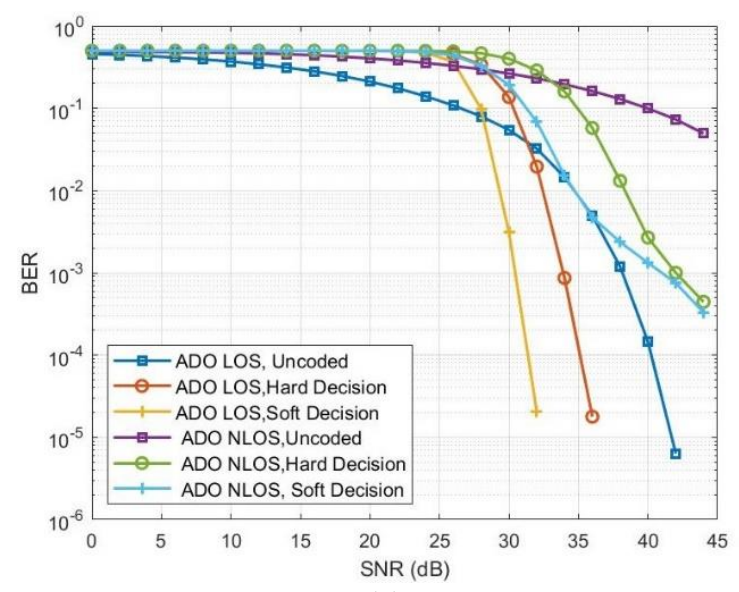

(c)

Figure. 9 BER performance of uncoded and coded of different O-OFDM for 256QAM modulation: (a) DCO-OFDM, (b) ACO-OFDM, and (c) ADO-OFDM 
Received: April 22, 2021. Revised: May 11, 2021.

Table 1. The BER performance comparisons for the examination O-OFDM in 256QAM modulation

\begin{tabular}{|c|c|c|c|c|c|c|}
\hline & \multicolumn{2}{|c|}{$\begin{array}{l}\text { DCO-OFDM } \\
\text { SNR in dB for }\end{array}$} & \multicolumn{2}{|c|}{$\begin{array}{l}\text { ACO-OFDM } \\
\text { SNR in dB for }\end{array}$} & \multicolumn{2}{|c|}{$\begin{array}{c}\text { ADO-OFDM } \\
\text { SNR in dB for }\end{array}$} \\
\hline Parameter & $\mathrm{BER}=10^{-3}$ & $\mathrm{BER}=10^{-5}$ & $\mathrm{BER}=10^{-3}$ & $\mathrm{BER}=10^{-5}$ & $\mathrm{BER}=10^{-3}$ & $\mathrm{BER}=10^{-5}$ \\
\hline \multicolumn{7}{|c|}{ Case 1: LOS (LED linear not use Rappe's model) } \\
\hline uncoded [16] & 36 & 39 & 23 & 25 & 37 & 40 \\
\hline hard-decision & 33 & 37 & 19 & 21 & 33 & 36 \\
\hline soft-decision & 31 & 33 & 13 & 17 & 29 & 31 \\
\hline \multicolumn{7}{|c|}{ Case 2: LOS (LED use Rappe's model, k=2) } \\
\hline uncoded [16] & $\mathrm{BER}=0$ & at $\mathrm{SNR}=45$ & 22.5 & 25.5 & 38 & 41 \\
\hline hard-decision & 37 & 41 & 19 & 21 & 34 & 36 \\
\hline soft-decision & 30.5 & 33 & 14 & 15 & 31 & 33 \\
\hline \multicolumn{7}{|c|}{ Case 3: NLOS (LED linear not use Rappe's model) } \\
\hline Uncoded [16] & \multicolumn{2}{|c|}{$\mathrm{BER}=0.09$ at $\mathrm{SNR}=45$} & 35 & 44 & \multicolumn{2}{|c|}{$\mathrm{BER}=0.05$ at $\mathrm{SNR}=45$} \\
\hline hard-decision & 43 & 45 & 27 & 33.5 & 41 & 44 \\
\hline soft-decision & 42 & 45 & 26 & 31 & 40 & 44 \\
\hline \multicolumn{7}{|c|}{ Case 4: NLOS (LED use Rappe's model, $\mathrm{k}=2$ ) } \\
\hline Uncoded [16] & \multicolumn{2}{|c|}{$\mathrm{BER}=0.02$ at $\mathrm{SNR}=45$} & 35 & 45 & \multicolumn{2}{|c|}{$\mathrm{BER}=0.05$ at $\mathrm{SNR}=45$} \\
\hline hard-decision & 45 & - & 27 & 33 & 42 & 45 \\
\hline soft-decision & 43 & 45 & 27 & 31.5 & 40.5 & 44.5 \\
\hline
\end{tabular}

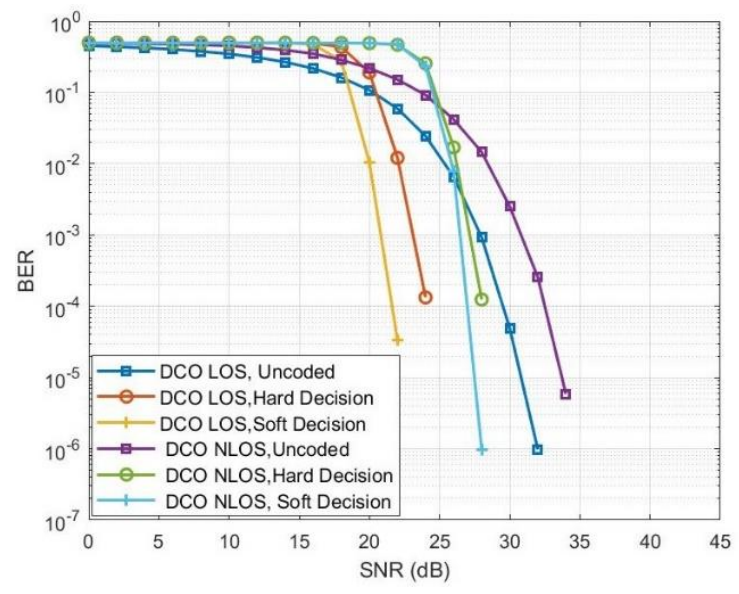

(a)

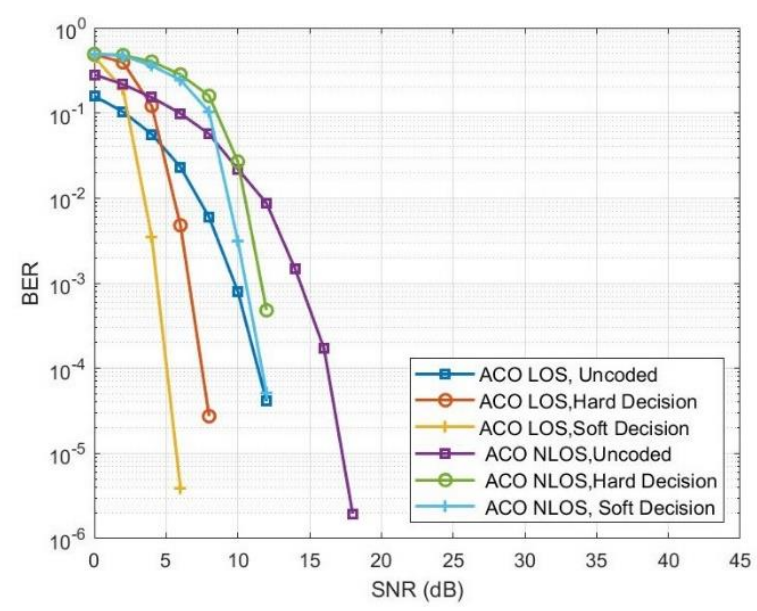

(b)

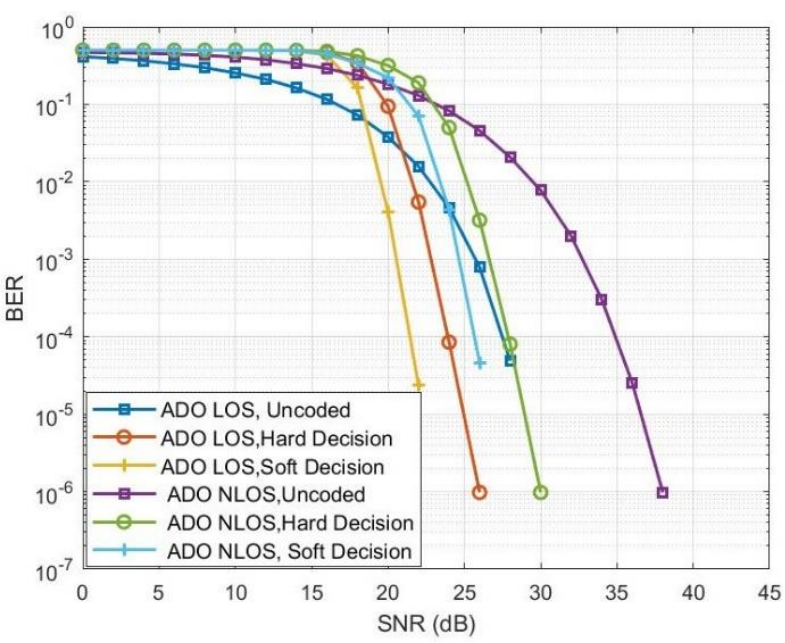

(c)

Figure.10 BER performance of uncoded and coded of different O-OFDM for QAM modulation:

(a) DCO-OFDM, (b) ACO-OFDM, and (c) ADO-OFDM 


\section{Conclusion}

This paper has investigated the enhancement of BER performance for DCO-OFDM, ACO-OFDM, and ADO-OFDM using the conVolutional encoder and Viterbi decoder. The main influencing performance parameters for VLC systems have been illustrated, such as channel types LOS or NLOS, SNR requirement, modulation constellation size, OOFDM schemes type, and non-linear LED behavior. The simulation results show the BER variation concerning the knee factor. So, for ADO-OFDM, LOS, 256QAM modulation $\mathrm{SNR}=38 \mathrm{~dB}$, the BER values for linear behavior and knee factors 50,10, and 2 respectively are $0.0007,0.0076,0.02$, and 0.3 . The proposed BER enhancement model utilizes the (3, 2, 7) conVolutional encoder and Viterbi algorithm for hard and soft decisions with O-OFDM VLC systems. Viterbi Algorithm offers an enhanced BER by finding likelihood for each bit based on minimum decision distance between transmitted and received signals. Simulations have been mentioned to evaluate the O-OFDM systems with/without LED's nonlinearity for LOS and NLOS channels. The proposed system results for different O-OFDM give smaller BER values than the uncoded response, and it helps to mitigate the LED's non-linearity and signal distortion. In ADO-OFDM, LOS, knee factor $=2 \mathrm{~m}$, the proposed model-based soft-decision can enhance BER from 0.015 to $2.3 \times 10^{-5}$ at $\mathrm{SNR}=22 \mathrm{~dB}$ for QAM. However, for NLOS, the proposed model reduces BER from 0.045 to $8.5 \times 10^{-5}$ at $\mathrm{SNR}=26 \mathrm{~dB}$. The ZFE and block-type channel estimator have been used with the NLOS model for the diffused channel. The estimator has tuned to evaluate the channel's frequency response several times to equalize the channel variations and the LED non-linearity. Also, simulation results indicated better performance for the soft-decision decoding, about $5 \mathrm{~dB}$ compared to hard-decision. Comparing the examined O-OFDM for QAM and 256QAM modulation over LOS or NLOS channel coded/uncoded system appears the BER values are low for large value of SNR. However, the BER for coded O-OFDM is better than in uncoded, and if increasing the modulation constellation size, the BER performance degrades. The BER ACO-OFDM is the best because it does not use DC bias that introduces clipping noise which degrades the performance. Utilizing the proposed model with ADO-OFDM shows the finer performance than DCO-OFDM. We will implement the examinated O-OFDM VLC systems in hardware for the actual case using Field Programmable Gate Arrays (FPGA) for upcoming research.

\section{Conflicts of Interest}

The authors declare no conflict of interest

\section{Author Contributions}

Conceptualization, Sinan M. Abdulsatar, Atheer A. Sabri, and Samir M. Hameed; methodology, software, validation, investigation, writing - original draft preparation, writing - review and editing, Samir M. Hameed; formal analysis, Atheer A. Sabri; supervision, project administration, Sinan M. Abdulsatar.

\section{References}

[1] S. Ariyanti and M. Suryanegara, "Visible light communication (VLC) for $6 \mathrm{G}$ techNo.logy: The potency and research challenges", In: Proc. World Conf. Smart Trends Syst. Secur. Sustain. WS4 2020, No. Vlc, pp. 490-493, 2020.

[2] M. A. Yaseen, A. K. Abass, and S. M. Abdulsatar, "Improving of Wavelength Division Multiplexing Based on Free Space Optical Communication via Power Comparative System", Wirel. Pers. Commun., No. 0123456789, 2021.

[3] E. Strinati, S. Barbarossa, J. Gonzalez-Jimenez, D. Kténas, N. Cassiau, L. Maret, and C. Dehos, "6G: The Next Frontier: From Holographic Messaging to Artificial Intelligence Using Subterahertz and Visible Light Communication", IEEE Veh. TechNo.l. Mag., Vol. 14, No. 3, pp. 42-58, 2019.

[4] M. A. Yaseen, A. K. Abass, and S. M. Abdulsatar, "Enhancing of multiwavelength free space optical communication system via optimizing the transceiver design parameters", Opt. Quantum Electron., Vol. 52, No. 8, pp. 110, 2020.

[5] M. M. Céspedes, B. G. Guzmán, and V. P. Gil Jiménez, "Lights and shadows: A comprehensive survey on cooperative and precoding schemes to overcome LOS blockage and interference in indoor VLC", Sensors (Switzerland), Vol. 21, No. 3, pp. 1-41, 2021.

[6] A. Assabir, J. Elmhamdi, and A. Hammouch, "Throughput enhancement of the edge user equipments based on the power-bandwidth tradeoff in the optical attocell networks", Int. J. Intell. Eng. Syst., Vol. 13, No. 5, pp. 337-355, 2020.

[7] N. Sharan and S. K. Ghorai, "PAPR reduction and No.n-linearity alleviation using hybrid of precoding and companding in a visible light communication (VLC) system", Opt. Quantum 
Electron., Vol. 52, No. 6, 2020.

[8] L. Hao, D. Wang, W. Cheng, J. Li, and A. Ma, "Performance enhancement of ACO-OFDMbased VLC systems using a hybrid autoencoder scheme", Opt. Commun., Vol. 442, No. March, pp. 110-116, 2019.

[9] S. M. Abdulsatar, "Peak to average power ratio reduction for coherent optical OFDM systems using phase rotatioNo.f the sub-carriers", Int. $J$. Intell. Eng. Syst., Vol. 13, No. 3, pp. 69-79, 2020.

[10] S. E. D. N. Mohamed, A. E. N. A. Mohamed, F. E. A. El-Samie, and A. N. Z. Rashed, "Performance enhancement of IM/DD optical wireless systems", Photonic Netw. Commun., Vol. 36, No. 1, pp. 114-127, 2018.

[11] X. Huang, F. Yang, X. Liu, H. Zhang, J. Ye, and J. Song, "Subcarrier and power allocations for dimmable enhanced ADO-OFDM with iterative interference cancellation", IEEE Access, Vol. 7, pp. 28422-28435, 2019.

[12] V. Maddala and R. R. Katta, "Adaptive ABC algorithm based PTS scheme for PAPR reduction in MIMOOFDM", Int. J. Intell. Eng. Syst., Vol. 10, No. 2, pp. 48-57, 2017.

[13] S. D. Dissanayake, S. Member, J. Armstrong, and S. Member, "ComparisoNo.f ACO-OFDM, DCO-OFDM and ADO-OFDM in IM / DD Systems", Journal of Lightwave TechNology, Vol. 31, No. 7, pp. 1063-1072, 2013.

[14] Z. Wang, T. Mao, and Q. Wang, "Optical OFDM for visible light communications", 2017 13th Int. Wirel. Commun. Mob. Comput. Conf. IWCMC 2017, No. 1, pp. 1190-1194, 2017.

[15] M. A. E. A. Eltoukhi, M. Abd-Elnaby, S. A. ElDolil, and F. E. A. El-Samie, "Efficient coding techniques for ADO-OFDM in IM/DD systems", Photonic Netw. Commun., Vol. 36, No. 1, pp. 128-139, 2018.

[16] P. P. Jativa, C. A. Azurdia-Meza, M. R. Canizares, D. Zabala-Blanco, and S. MontejoSanchez, "Performance Analysis of OFDMBased VLC Schemes in NLOS Channels", 2020 South Am. Colloq. Visible Light Commun. SACVC 2020 - Proc., 2020.

[17] Y. Chen, Z. Li, P. Liu, and S. Liu, "The BER performance comparisoNo.f MSK and GMSK schemes for short-range visible light communication", 2017 9th IEEE Int. Conf. Commun. Softw. Networks, ICCSN 2017, Vol. 2017-Janua, pp. 611-614, 2017.

[18] H. Elgala, R. Mesleh, and H. Haas, "An led model for intensity-modulated optical communication systems", IEEE Photonics TechNo.l. Lett., Vol. 22, No. 11, pp. 835-837, 2010.
[19] H. Lu, J. Jin, and J. Wang, "AlleviatioNo.f led No.nlinearity impact in visible light communication using companding and predistortion", IET Commun., Vol. 13, No. 7, pp. 818-821, 2019.

[20] A. A. Abdulkafi, M. Y. Alias, and Y. S. Hussein, "Performance analysis of DCO-OFDM in VLC system”, 2015 IEEE 12th Malaysia Int. Conf. Commun. MICC 2015, Vol. 1, No. Micc, pp. 163-168, 2016.

[21] R. Mesleh, H. Elgala, and H. Haas, "LED No.nlinearity mitigation techniques iNo.ptical wireless OFDM communication systems", $J$. Opt. Commun. Netw., Vol. 4, No. 11, pp. 865875, 2012.

[22] S. Kumar and P. Singh, "Filter Bank Multicarrier Modulation Schemes for Visible Light Communication", Wirel. Pers. Commun., Vol. 113, No. 4, pp. 2709-2722, 2020.

[23] J. Armstrong, "OFDM for optical communications", J. Light. TechNo.l., Vol. 27, No. 3, pp. 189-204, 2009.

[24] W. Hong, T. Li, W. Li, and X. Shi, "Analysis of DCO-OFDM for Indoor Visible Light Communications," J. Phys. Conf. Ser., Vol. 1606, No. 1, p. 012005, 2020.

[25] L. Deng, Y. Fan, and Q. Zhao, "A No.vel PAPR reduction scheme for VLC DCO-OFDM systems", Opt. Commun., Vol. 426, No. May, pp. 164-169, 2018.

[26] J. S. Sheu, B. J. Li, and J. K. Lain, "LED No.nlinearity mitigation techniques for optical OFDM-based visible light communications", IET Optoelectron., Vol. 11, No. 6, pp. 259-264, 2017.

[27] M. A. Abu-Rgheff, Introduction to Digital Communications. 2007.

[28] A. J. Viterbi and A. J. Viterbi, "ConVolutional Codes and Their Performance in Communication Systems", IEEE Trans. Commun. TechNo.l., Vol. 19, No. 5, pp. 751-772, 1971.

[29] T. Adiono, Y. Aska, S. Fuada, and A. A. Purwita, "DesigNo.f aNo.FDM System for VLC with a Viterbi Decoder", IEIE Trans. Smart Process. Comput., Vol. 6, No. 6, pp. 455-465, 2017.

[30] M. A. Fleah and Q. F. Al-Doori, "Design and ImplementatioNo.f Turbo encoder/decoder using FPGA", 1st Int. Sci. Conf. Comput. Appl. Sci. CAS 2019, pp. 46-51, 2019.

[31] Y. S. Hussein, M. Y. Alias, and A. A. Abdulkafi, "On performance analysis of LS and MMSE for channel estimation in VLC systems", Proceeding - 2016 IEEE 12th Int. Colloq. Signal Process. its Appl. CSPA 2016, No. March, pp. 
204-209, 2016.

[32] O. Şayli, H. Doğan, and E. Panayirci, "On channel estimation in DC biased optical OFDM systems over VLC channels", Int. Conf. Adv. TechNo.l. Commun., No. 1, pp. 147-151, 2016. 